\title{
Thirty years and 1663 consecutive Norwood procedures: Has survival plateaued?
}

Christopher E. Mascio, MD, ${ }^{a}$ Mallory L. Irons, MD, MBE, ${ }^{b}$ Richard F. Ittenbach, $\mathrm{PhD},{ }^{c}$

J. William Gaynor, MD, ${ }^{\text {a }}$ Stephanie M. Fuller, MD, ${ }^{a}$ Michelle Kaplinski, MD, ${ }^{\mathrm{d}}$ Andrea T. Kennedy, BS, James M. Steven, MD, ${ }^{\mathrm{f}}$ Susan C. Nicolson, $\mathrm{MD},{ }^{\mathrm{f}}$ and Thomas L. Spray, $\mathrm{MD}^{\mathrm{a}}$

\section{ABSTRACT}

Objective: Hypoplastic left heart syndrome is one of the most common and challenging lesions requiring surgical intervention in the neonatal period. The Norwood procedure for hypoplastic left heart syndrome was first reported in 1983. The objective of this study was to describe early outcomes after the Norwood procedure at a single institution over 30 years.

Methods: This retrospective cohort study included all patients with hypoplastic left heart syndrome (and variants) who underwent the Norwood procedure between January 1984 and May 2014 at a single institution. The study period was divided into 6 eras: era 1, 1984 to 1988 ; era 2, 1989 to 1993; era 3, 1994 to 1998; era 4, 1999 to 2003; era 5, 2004 to 2008; and era 6, 2009 to 2014. The primary outcome was in-hospital mortality after the Norwood procedure. Binomial point estimates complete with $95 \%$ confidence intervals $\left(\mathrm{CL}_{0.95}\right)$ were computed for the entire cohort and by era.

Results: During the study period, 1663 infants underwent the Norwood procedure. Overall in-hospital mortality was $25.9 \%\left(\mathrm{CL}_{0.95}, 23.8-28.0\right)$. Mortality by chronologic era was 40.4\% $\left(\mathrm{CL}_{0.95}, 34.9-45.9\right), 33.6 \%\left(\mathrm{CL}_{0.95}, 29.2-37.9\right)$, $28.7 \% \quad\left(\mathrm{CL}_{0.95}, 22.8-34.6\right), 14.9 \%\left(\mathrm{CL}_{0.95}, 10.4-19.3\right), 11.2 \% \quad\left(\mathrm{CL}_{0.95}, 7.4-\right.$ $15.0)$, and $15.7 \%\left(\mathrm{CL}_{0.95}, 10.3-21.1\right)$. Survival was improved in eras 4 to 6 compared with eras 1 to $3(P$ all $<.03)$. Anomalous pulmonary drainage, moderate to severe atrioventricular valve regurgitation, lower birth weight, earlier era, younger gestational age, genetic anomaly, preterm birth, race other than white or African-American, and lower weight at the Norwood procedure were associated with increased mortality. Mortality was greatest in patients with 3 or more risk factors. In the best-fitting multiple covariate model, anomalous pulmonary venous drainage, gestational age in weeks, genetic anomaly, and race other than white and African American were statistically significant contributors, after adjusting for era.

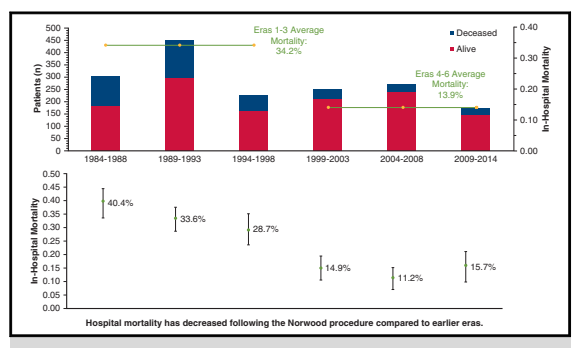

Hospital mortality has decreased after the Norwood procedure compared with earlier eras.

\section{Central Message}

Hospital mortality after the Norwood procedure has decreased in recent eras. We identified patient-specific factors that affect mortality. $\mathrm{Pa}$ tients with 3 or more risk factors have worse survival.

\section{Perspective}

The operative technique of the Norwood procedure today is nearly identical to the original description. Hospital mortality for patients undergoing this procedure has decreased in recent eras, but it remains unchanged for the last 15 years. Patient-specific risk factors continue to play a key role in survival. Lower-risk patients have lower mortality rates compared with their high-risk counterparts.

See Commentaries on pages 230 and 232.

Conclusions: Survival after the Norwood procedure has plateaued despite improvements in diagnosis, perioperative care, and surgical techniques. Nonmodifiable patient characteristics are important determinants of the risk of mortality. (J Thorac Cardiovasc Surg 2019;158:220-9)

From the ${ }^{\mathrm{a}}$ Division of Cardiothoracic Surgery, Department of Surgery, ${ }^{\mathrm{d}}$ Division of Cardiology, Department of Pediatrics, ${ }^{e}$ Clinical Data Analytics, Office of Clinical Quality Improvement, and ${ }^{\mathrm{f}}$ Division of Cardiothoracic Anesthesiology, Department of Anesthesiology and Critical Care Medicine, Children's Hospital of Philadelphia, Philadelphia, Pa; ${ }^{\mathrm{b}}$ Division of Cardiovascular Surgery, Department of Surgery, University of Pennsylvania, Philadelphia, Pa; and ${ }^{\mathrm{c}}$ Division of Biostatistics and Epidemiology, Department of Pediatrics, Cincinnati Children's Hospital, Cincinnati, Ohio.

Supported by the Daniel M. Tabas and Alice Langdon Warner Endowed Chairs in Pediatric Cardiothoracic Surgery.

Date and number of Institutional Review Board approval: June 25, 2014; 14-010817.
Received for publication April 28, 2018; revisions received Nov 26, 2018; accepted for publication Dec 27, 2018.

Address for reprints: Christopher E. Mascio, MD, Pediatric Cardiothoracic Surgery, Children's Hospital of Philadelphia, Perelman School of Medicine, University of Pennsylvania, 3401 Civic Center Boulevard, Suite 12NW10, Philadelphia, PA 19104 (E-mail: mascioc1@email.chop.edu).

0022-5223/\$36.00

Copyright (C) 2019 Published by Elsevier Inc. on behalf of The American Association for Thoracic Surgery

https://doi.org/10.1016/j.jtcvs.2018.12.117 


\section{Abbreviations and Acronyms \\ $\mathrm{CL}_{0.95}=95 \%$ confidence interval \\ $\mathrm{ECMO}=$ extracorporeal membrane oxygenation \\ HLHS $=$ hypoplastic left heart syndrome}

To view the AATS Annual Meeting Webcast, see the URL next to the webcast thumbnail.

Aortic atresia or stenosis along with mitral atresia or stenosis and hypoplasia of the left ventricle were initially described by Lev in $1952^{1}$ and referred to as "hypoplasia of the aortic tract complexes" with the term "hypoplastic left heart syndrome" (HLHS) coined in 1958 by Noonan and Nadas. ${ }^{2}$ HLHS is one of the most common lesions requiring surgical correction in the neonatal period, and without surgical intervention, this lesion is uniformly lethal. The first report of an attempted palliative operation for HLHS was in 1977 by Doty and Knott. ${ }^{3}$ All of the patients described died of poor right ventricular function or coronary ischemia. In 1983, Norwood and associates ${ }^{4}$ were the first to report success with the palliative procedure that is still performed today. The important components of this procedure include amalgamation of the main pulmonary artery to the ascending aorta to provide unobstructed systemic ventricle outflow, atrial septectomy to allow unobstructed pulmonary venous drainage, and a systemic to pulmonary shunt as a source of controlled pulmonary blood flow. ${ }^{4}$ Since Norwood and colleagues' first description, many advances have been made in fetal diagnosis, preoperative stabilization, operative and anesthetic techniques, and postoperative care of patients with HLHS. Perioperative morbidity and mortality have improved since the first report of successful palliation, but remains high compared with other forms of congenital heart disease. Our institution has a long and significant experience with the Norwood procedure. In this study, we investigated changes in hospital mortality over time and the impact of patient-specific risk factors on mortality.

\section{PATIENTS AND METHODS}

The primary objectives of this retrospective cohort study were to examine hospital mortality and the impact of patient-specific risk factors on in-hospital mortality after the Norwood procedure from January 1, 1984, to May 6, 2014. All subjects who underwent a Norwood procedure between these dates were included (HLHS and all variants). There were no exclusion criteria. Data on patient characteristics were abstracted from paper charts and electronic medical records (Table 1).
TABLE 1. Patient characteristics $(N=1663)$

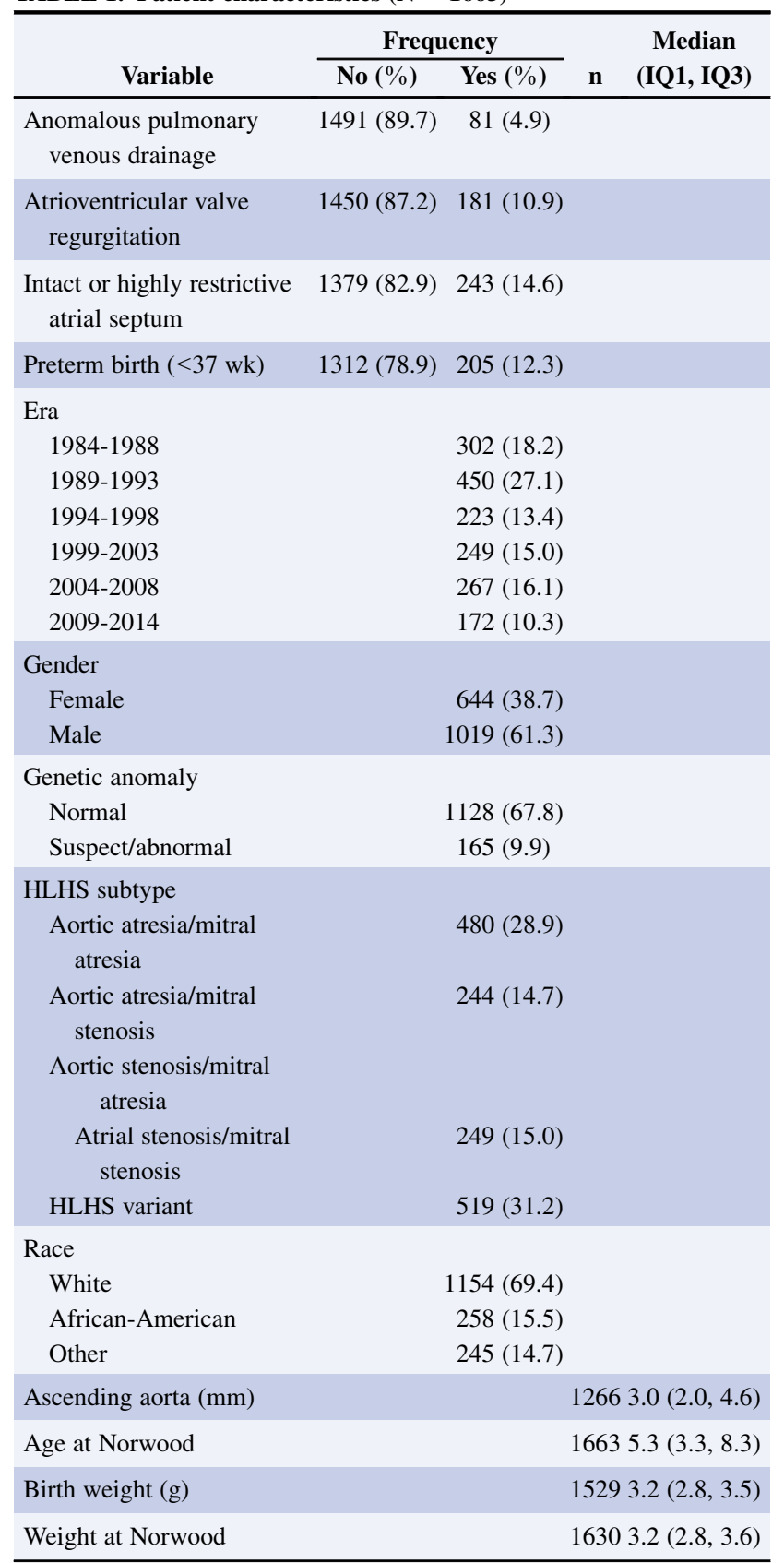

$I Q$, Interquartile; $H L H S$, hypoplastic left heart syndrome.

\section{Analysis Plan}

Data analysis proceeded in 3 distinct phases: a descriptive phase in which we computed descriptive statistics for the entire cohort of 1663 infants and children undergoing cardiac surgery for HLHS and its variants; a mortality phase in which we estimated prevalence of mortality for the entire cohort as well as by era; and a risk modeling phase in which we sought to identify risk factors for in-hospital mortality among the cohort. The total number of risk factors by patient were computed and compared across eras. All data were analyzed using SAS v9.4 (SAS Institute Inc, Cary, NC). 
TABLE 2. Single and multiple covariate risk factor models for in-hospital mortality, 1984 to $2014(\mathrm{~N}=1663)$

\begin{tabular}{|c|c|c|c|c|c|c|c|}
\hline \multirow[b]{2}{*}{ Potential risk factors } & \multicolumn{4}{|c|}{$\begin{array}{l}\text { Single covariate models* } \\
\text { adjusted for era }\end{array}$} & \multicolumn{3}{|c|}{$\begin{array}{l}\text { Best fitting multiple covariate } \\
\text { model adjusted for era }\end{array}$} \\
\hline & $\mathbf{n}$ & $\beta$ (SEM) & OR $\left(\mathbf{C I}_{\mathbf{0 . 9 5}}\right)$ & $P$ value & $\beta$ (SEM) & OR $\left(\mathbf{C I}_{0.95}\right)$ & $P$ value \\
\hline Ascending aorta $(\mathrm{mm})$ & 1266 & $-0.07(0.04)$ & $0.94(0.86-1.01)$ & .11 & & & \\
\hline Age at Norwood (d) & 1663 & $0.00(0.00)$ & $1.00(1.00-1.01)$ & .21 & & & \\
\hline Anomalous pulmonary veins (reference: none) & 1572 & $0.38(0.12)$ & $2.12(1.32-3.39)$ & $<.01$ & $0.36(0.16)$ & $2.05(1.11-3.80)$ & .02 \\
\hline Atrioventricular valve regurgitation (reference: none) & 1631 & $0.25(0.09)$ & $1.65(1.17-2.34)$ & $<.01$ & & & \\
\hline Birth weight $(\mathrm{kg})$ & 1529 & $-0.83(0.11)$ & $0.43(0.35-0.54)$ & $<.01$ & & & \\
\hline Era (reference: 1984-1988) & 1663 & & & $<.01$ & & & $<.01$ \\
\hline $1989-1993$ & & $0.56(0.10)$ & $0.74(0.55-1.01)$ & $<.01$ & $0.40(0.17)$ & $0.36(0.23-0.58)$ & .02 \\
\hline 1994-1998 & & $0.34(0.14)$ & $0.59(0.41-0.86)$ & .01 & $0.17(0.20)$ & $0.29(0.16-0.50)$ & .40 \\
\hline $1999-2003$ & & $-0.50(0.16)$ & $0.26(0.17-0.39)$ & $<.01$ & $-0.58(0.18)$ & $0.14(0.08-0.22)$ & $<.01$ \\
\hline 2004-2008 & & $-0.82(0.17)$ & $0.19(0.12-0.29)$ & $<.01$ & $-0.91(0.19)$ & $0.10(0.06-0.17)$ & $<.01$ \\
\hline $2009-2014$ & & $-0.44(0.18)$ & $0.28(0.17-0.44)$ & .02 & $-0.50(0.20)$ & $0.15(0.08-0.26)$ & .01 \\
\hline Gestational age (wk) & 1517 & $-0.23(0.03)$ & $0.79(0.74-0.84)$ & $<.01$ & $-0.25(0.04)$ & $0.78(0.72-0.84)$ & $<.01$ \\
\hline Gender (reference: male) & 1663 & $0.12(0.06)$ & $1.26(1.00-1.59)$ & .05 & & & \\
\hline Genetic anomaly (reference: none) & 1293 & $0.33(0.10)$ & $1.93(1.32-2.82)$ & $<.01$ & $0.23(0.11)$ & $1.59(1.05-2.42)$ & .03 \\
\hline HLHS subtype (reference: aortic atresia/mitral atresia) & 1492 & & & .27 & & & \\
\hline Aortic atresia/mitral stenosis & & $0.06(0.12)$ & $0.96(0.67-1.38)$ & .60 & & & \\
\hline Aortic stenosis/mitral atresia and & & & & & & & \\
\hline Aortic stenosis/mitral stenosis & & $-0.25(0.13)$ & $0.70(0.48-1.02)$ & .05 & & & \\
\hline Variant & & $0.09(0.10)$ & $0.98(0.74-1.32)$ & .38 & & & \\
\hline Intact atrial septum (reference: No.) & 1622 & $0.15(0.08)$ & $1.34(0.99-1.82)$ & .06 & & & \\
\hline Preterm birth (kg, reference: No.) & 1517 & $0.58(0.08)$ & $3.20(2.31-4.44)$ & $<.01$ & & & \\
\hline Race (reference: white) & 1657 & & & $<0.01$ & & & $<0.01$ \\
\hline African American & & $-0.02(0.11)$ & $1.56(1.13-2.16)$ & .83 & $-0.08(0.15)$ & $1.54(0.99-2.38)$ & .57 \\
\hline Other & & $0.49(0.11)$ & $2.62(1.89-3.64)$ & $<.01$ & $0.60(0.14)$ & $3.03(2.02-4.54)$ & $<.01$ \\
\hline Weight at stage 1 surgery & 1630 & $-0.57(0.10)$ & $0.57(0.47-0.68)$ & $<.01$ & & & \\
\hline Intercept & 1191 & & & & $8.92(1.44)$ & & \\
\hline
\end{tabular}

$\overline{S E M}$, Standard error of the mean; $O R$, odds ratio; $C I_{0.95}, 95 \%$ confidence interval; $H L H S$, hypoplastic left heart syndrome. *All single covariate models were adjusted for era; however, era was also tested individually as a categoric covariate with no additional adjustments.

\section{Descriptive Phase}

Descriptive statistics were computed for the entire sample of patients using both parametric and nonparametric measures of central tendency, variability, and association. Additional "flag" variables were created, one for each of the original variables in the data set, to track and evaluate missingness over the 30-year study period. Patterns of missingness were analyzed for the data set as a whole and by era. For purposes of this study, eras were divided as follows: 1983 to 1988 (era 1), 1989 to 1993 (era 2), 1994 to 1998 (era 3), 1999 to 2003 (era 4), 2004 to 2008 (era 5), and 2009 to 2014 (era 6), primarily to balance out the groups and to allow for potential differences in staffing and practice patterns. Completeness of data for variables used in our final model, across all eras, was $94.4 \%$ and ranged from a low of $44 \%$ for 2 to a high of $100 \%$ in eras 5 and 6 . We had no missing data for our outcome (mortality) or for our adjusting covariate (era). A total of 1191 of the 1663 cases were used for complete case modeling. Examination of missingness patterns yielded no discernable trends due to patient characteristics across eras or conditions, instead being due to variations in documentation, practice patterns, and the evolution of national and international registries.

\section{Mortality Phase}

Mortality rates were computed for hospital discharge status (alive vs deceased) using standard binomial proportions, complete with $95 \%$ confidence intervals. This was done initially for the entire cohort and then individually by era. Rates across eras were then compared using an ordered logistic regression model with Tukey-Kramer adjustments for multiple comparisons. As a follow-up to the former, mortality rates were also computed for each of the 5 HLHS subtypes across the entire cohort for descriptive purposes only.

\section{Risk Modeling Phase}

In the risk modeling phase, 3 sets of logistic regression models were specified and tested. First, a single logistic regression model was tested with era to get a sense of the relationship between mortality at discharge and era. Second, 14 logistic regression models were specified and tested, each with a single testable covariate adjusted for era. Covariates used in these models were limited to patient-related factors, examples of which include ascending aorta diameter, age at Norwood, birth weight, gestational age, and weight at stage 1 surgery (Table 2 shows a complete listing of covariates used). Finally, a single, best-fitting, multiple covariate model was specified and tested for significance, after adjusting for era. Single covariate terms with $P$ values less than .10 were considered for inclusion in the final, best-fitting multiple covariate model. A final, follow-up analysis was completed as a complement to the risk modeling phase in which we compared mortality rates between those who had 2 or fewer risk factors and those who had 3 or more risk factors, using a 2- 
TABLE 3. Frequency and mortality of anatomic subtypes undergoing the Norwood procedure

\begin{tabular}{lcc}
\hline \multicolumn{1}{c}{ Anatomy } & Frequency & Mortality \\
\hline HLHS variant & $34.8 \%(519 / 1492)$ & $26.8 \%$ \\
Aortic atresia/mitral atresia & $32.2 \%(480 / 1492)$ & $26.7 \%$ \\
\hline Aortic atresia/mitral stenosis & $16.4 \%(244 / 1492)$ & $27.0 \%$ \\
Aortic stenosis/mitral stenosis & $15.4 \%(230 / 1492)$ & $20.4 \%$ \\
Aortic stenosis/mitral atresia & $1.27 \%(19 / 1492)$ & $31.6 \%$ \\
\hline
\end{tabular}

$H L H S$, Hypoplastic left heart syndrome.

sample test of proportion. Risk factors used as indicators for this analysis included the presence of anomalous pulmonary venous drainage, moderate to severe atrioventricular valve regurgitation, confirmed or suspected genetic anomaly, presence of an intact or highly restrictive atrial septum, preterm birth (dichotomized at gestational age $>37$ weeks), and weight at stage 1 reconstruction (dichotomized at $>2.5 \mathrm{~kg}$ ). All models tested conducted were evaluated using model- and term-specific Wald (chi-square) statistics at the unadjusted $\alpha=0.05$ level. Finally, mortality rates were compared for eras with 0 to 2 risk factors compared with 3 or more risk factors.

\section{RESULTS}

From January 1, 1984, to May 6, 2014, 1663 Norwood procedures were performed at our institution. The median weight (interquartile range) and age at the time of the Norwood procedure were $3.2(2.8-3.6) \mathrm{kg}$ and 5.3 (3.3-8.3) days, respectively. Median gestational age was 39.0 (38.0$39.0)$ weeks. Preterm birth ( $<37$ weeks gestation) was present in $13.5 \%(\mathrm{n}=205 / 1517)$. Male patients comprised $61.3 \%(\mathrm{n}=1019 / 1663)$ of all patients. The most common race was white $(69.6 \%, \mathrm{n}=1154 / 1657)$. Genetic anomalies were suspected or confirmed in $12.8 \%(n=165 / 1293)$. The Norwood procedure was performed most commonly for HLHS variants $(34.8 \%, 519 / 1492)$, and the most prevalent HLHS subtype was aortic atresia/mitral atresia $(32.2 \%$, 480/1492). The median size of the ascending aorta was $3.0(2.0,4.6) \mathrm{mm}$ by preoperative echocardiographic assessment. Anomalous pulmonary venous return was present in 5.2\% $(n=81 / 1572)$ of patients. Preoperative moderate or severe atrioventricular valve regurgitation was present in $11.1 \%(\mathrm{n}=181 / 1631)$. An intact or highly restrictive atrial septum was present in $15.0 \%(n=243 /$ 1622) (Table 1).

TABLE 4. Mortality by era

\begin{tabular}{lcc}
\hline Era & Years & Mortality \\
\hline 1 & $1984-1988$ & $40.4 \%$ \\
2 & $1989-1993$ & $33.6 \%$ \\
3 & $1994-1998$ & $28.7 \%$ \\
4 & $1999-2003$ & $14.9 \% *$ \\
5 & $2004-2008$ & $11.2 \% *$ \\
6 & $2009-2013$ & $15.7 \% *$ \\
\hline$* P<.03$ compared with eras 1 to 3.
\end{tabular}

Overall in-hospital mortality for the 30-year period was $25.9 \%$ ( $\mathrm{n}=431 / 1663)$, with no substantive differences among HLHS subtypes (Table 3). As a follow-up to overall mortality, we divided the data into 6 eras of approximately 5 years each. It should be noted that era 6 was slightly longer than the others at 5 years and 4 months. Mortality rates for the 5 eras ranged from a high of $40.4 \%$ in era 1 (1984-1988) to a low of $11.2 \%$ in era 5 (2004-2008), with significantly lower mortality rates in eras 4,5 , and 6 compared with eras 1, 2, and 3 (all $P<.03$ ) (Figure 1 and Tables 4 and 5).

We evaluated the impact of patient-specific risk factors on mortality using logistic regression. For the variables included in the final analysis, overall data completeness was $94.4 \%$. In univariate modeling, the following factors were associated with an increased risk of operative mortality: anomalous pulmonary venous drainage, moderate or severe atrioventricular valve regurgitation, lower birth weight, earlier era of surgery, lower gestational age, presence or suspicion of genetic anomaly, preterm birth ( $<37$ weeks), race other than white or African-American, and lower weight at the time of surgery. Of note, ascending aorta diameter, age at surgery, and HLHS subtype were not associated with risk of mortality. In the best-fitting multiple covariate model, anomalous pulmonary venous drainage, gestational age in weeks, genetic anomaly, and race other than white and African American were statistically significant contributors after adjusting for era (Table 2).

Finally, we evaluated the impact of multiple patientspecific risk factors on mortality. Multiple risk factors per patient were increasingly identified with later era of surgery. In the earliest era, 3 or more risk factors were identified in $15.9 \%$ of patients. However, this proportion increased to $30.8 \%$ in the most recent era. Across all eras, mortality increased as the number of risk factors increased (Table 6). For the entire cohort, mortality for patients with 0 to 2 identified risk factors was $23.8 \%$ and increased to $32.6 \%$ for those with 3 or more risk factors $(P=.001)$. For example, in the latest era (2009-2014), mortality was $10.9 \%$ for patients with 0 to 2 risk factors and $26.4 \%$ for those with 3 or more identified risk factors.

\section{DISCUSSION}

Successful palliation for HLHS was first reported just over 3 decades ago. ${ }^{4}$ We describe early mortality for the largest single-institution cohort of patients undergoing the Norwood procedure. For the entire 30-year period, inhospital mortality was $25.9 \%$. Although operative mortality decreased over the first 15 years, survival has plateaued over the last 15 years. The decline in mortality seen over the early eras is likely multifactorial. Contributing factors likely include increasing experience with the operation, introduction of prenatal diagnosis, and improvements in perioperative care. 
TABLE 5. Comparison of mortality rates across eras $(\mathrm{N}=1663)$

\begin{tabular}{|c|c|c|c|c|c|c|}
\hline Era (mortality, y) & $\begin{array}{c}\text { Era 1 } \\
\mathbf{n}=\mathbf{3 0 2}\end{array}$ & $\begin{array}{c}\text { Era 2 } \\
n=450\end{array}$ & $\begin{array}{c}\text { Era 3 } \\
n=223\end{array}$ & $\begin{array}{c}\text { Era 4 } \\
n=249\end{array}$ & $\begin{array}{c}\text { Era 5 } \\
\mathbf{n}=172\end{array}$ & $\begin{array}{c}\text { Era } 6 \\
n=172\end{array}$ \\
\hline Era $1(40.4 \%, 1984-1988)$ & 一 & 0.40 & 0.06 & $<0.01$ & $<0.01$ & $<0.01$ \\
\hline Era $2(33.6 \%, 1989-1993)$ & & & 0.80 & $<0.01$ & $<0.01$ & $<0.01$ \\
\hline Era $3(28.7 \%, 1994-1998)$ & & & & $<0.01$ & $<0.01$ & 0.03 \\
\hline Era $4(14.9 \%, 1999-2003)$ & & & & & 0.83 & 1.00 \\
\hline Era $5(11.2 \%, 2004-2008)$ & & & & & & 0.76 \\
\hline Era $6(15.7 \%, 2009-2013)$ & & & & & & - \\
\hline
\end{tabular}

$P$ values presented are adjusted for all pairwise comparisons using the Tukey-Kramer adjustment for multiple comparisons.

In addition to analyzing trends in HLHS survival over time, we also examined the impact of patient-specific risk factors on mortality. By univariable analysis, many previously reported factors were associated with an increased risk of death, including anomalous pulmonary venous drainage, moderate to severe atrioventricular valve regurgitation, lower birth weight, younger gestational age, confirmed or suspected genetic anomaly, presence of a highly restrictive or intact atrial septum, and lower weight at surgery. Even in this large cohort, other factors including ascending aorta diameter, age at surgery, and HLHS subtype were not associated with increased mortality. By multivariate analysis, only 4 patient factors other than earlier era of operation (anomalous pulmonary venous drainage, gestational age, genetic anomaly, and race other than white or African-American) were associated with increased mortality.

We have also demonstrated that the presence of multiple risk factors in an individual patient is associated with higher mortality. The number of patients with multiple risk factors increased significantly in the more recent eras studied. In the earliest era, $84.1 \%$ of the patients had 0 to 2 identified risk factors. This proportion decreased to $69.2 \%$ in the latest era, when more patients had 3 or more identified risk factors. This change may be due to several factors; for example, in the earliest era, patients with multiple risk factors may have died before referral for surgery. In addition, it is conceivable that over the study period, a well-established institution such as ours may have seen a higher-risk population of referrals, as other institutions developed expertise with the Norwood procedure. Finally, despite our best efforts, there is a greater proportion of missing data in the early eras, particularly genetic information. Nonetheless, overall data completeness was more than $90 \%$, and ascertainment bias alone is unlikely to explain these changes. Mortality has decreased over the course of the study, and the benefit has been greatest in patients with fewer risk factors. However, although mortality decreased for the highestrisk patients, overall mortality still remains greater than
$20 \%$. The plateau in survival may be explained by the increasing proportion of high-risk patients, despite overall improving results with surgical technique. Importantly and unfortunately, the identified risk factors are not currently modifiable.

There have been many suggested modifications in technique to the Norwood procedure, such as method of arch reconstruction, reintroduction of the right ventricle to pulmonary artery conduit, and use of the hybrid procedure. However, none of these variations have significantly reduced hospital mortality. Results of the Pediatric Heart Network Single Ventricle Randomization trial have demonstrated no difference in hospital mortality between patients receiving a systemic artery to pulmonary artery shunt (known as a modified Blalock-Taussig) and patients receiving a right ventricle to pulmonary artery shunt. ${ }^{5}$ In this cohort of 549 patients, overall hospital mortality was $16 \%$ and independent risk factors for mortality included lower birth weight, genetic abnormality, extracorporeal membrane oxygenation (ECMO), and open sternum. It should be noted that the Single Ventricle Randomization trial did demonstrate differences in interstage survival and 1-year transplant-free survival depending on shunt type. However, this study only examined hospital survival and did not examine the interstage period or 1-year survival.

As an alternative to the Norwood procedure for stage 1 palliation, the hybrid procedure for HLHS has been adopted by some institutions. In a comparison of Norwood procedure and hybrid procedure outcomes, the Congenital Heart Surgeons' Society found that the hybrid approach did not provide a lower-risk alternative to the Norwood procedure. ${ }^{6}$ In a smaller series, Baba and colleagues ${ }^{7}$ also compared the hybrid procedure to the Norwood procedure and found no difference in hospital survival.

We have previously reported outcomes for some of the patients in this study, and the findings are similar. In 2000, Mahle and coworkers ${ }^{8}$ described our institution's initial 15-year experience with the Norwood procedure. At that time, mortality remained high with 1-, 2-, 5-, 10-, 

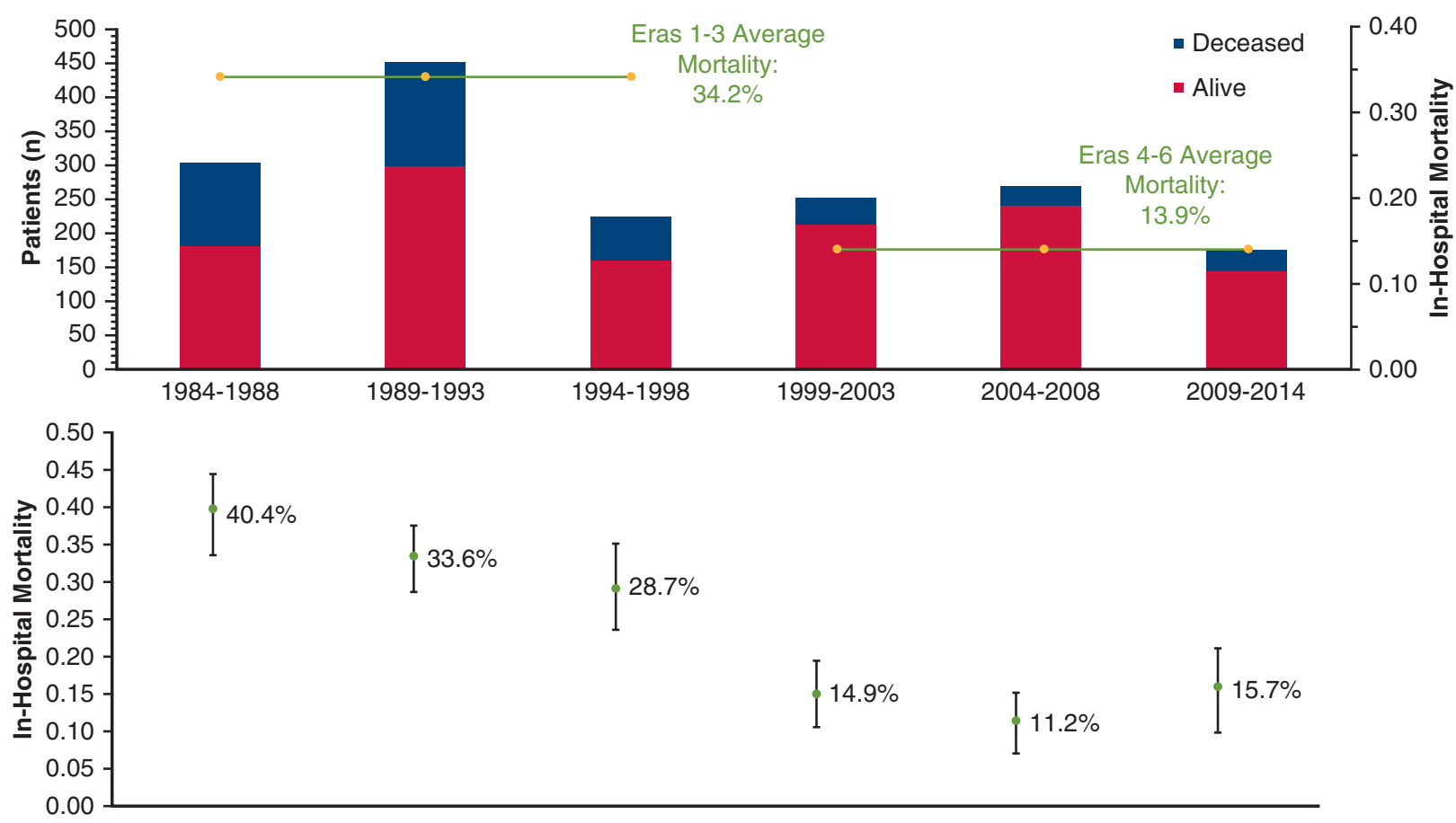

FIGURE 1. Hospital mortality has decreased after the Norwood procedure compared with earlier eras.

and 15 -year survivals of $51 \%, 43 \%, 40 \%, 39 \%$, and $39 \%$, respectively. Age at surgery of more than 14 days and weight at surgery of less than $2.5 \mathrm{~kg}$ were both associated with increased mortality. Later era of Norwood procedure was also associated with improved survival in that series. In 2002, Gaynor and colleagues ${ }^{9}$ described 158 patients undergoing the Norwood procedure over a 3.5-year period. As in the current report, patient characteristics were important determinants of operative mortality. Overall hospital survival in this cohort was $77 \%$, despite a patient population with low weight at the time of surgery or an associated anomaly (cardiac or extracardiac) present in approximately half of the patients $(46 \%)$ ). Operative survival for patients without these risk factors was $88 \%$ compared with $73 \%$ for those with 1 or more risk factors. There was no difference in mortality between HLHS patients and those with HLHS variants.

Other investigators have similar findings. In a series of 219 patients undergoing the Norwood procedure, Alsoufi and colleagues ${ }^{10}$ found postoperative ECMO, genetic or major extracardiac anomalies, unplanned cardiac reoperation, use of the modified Blalock-Taussig shunt, and prematurity as important risk factors for mortality. In a cohort of 28 patients weighing less than or equal to $2.5 \mathrm{~kg}$, Kalfa and colleagues $^{11}$ demonstrated that very low birth weight $(<1.5 \mathrm{~kg})$, delayed surgery, preoperative comorbidities, postoperative ECMO, neurologic complications, and dialysis were associated with higher hospital mortality. A 22-

TABLE 6. Mortality by total number of risk factors and era $(N=1663)$

\begin{tabular}{|c|c|c|c|c|}
\hline \multirow[b]{2}{*}{ Era (mortality, y) } & \multicolumn{4}{|c|}{ No. of identified risk factors per subject } \\
\hline & $\mathbf{0}$ & 1 & 2 & $\geq \mathbf{3}$ \\
\hline Era $1(40.4 \%, 1984-1988)$ & $59(39.3)$ & $18(47.4)$ & $18(30.0)$ & $22(46.8)$ \\
\hline Era $2(33.6 \%, 1989-1993)$ & $63(29.0)$ & $23(36.5)$ & $32(30.5)$ & $33(50.8)$ \\
\hline Era $3(28.7 \%, 1994-1998)$ & $27(25.2)$ & $6(24.0)$ & $15(31.2)$ & $16(37.2)$ \\
\hline Era $4(14.9 \%, 1999-2003)$ & $5(8.2)$ & $5(12.8)$ & $9(11.4)$ & $18(25.7)$ \\
\hline Era $5(11.2 \%, 2004-2008)$ & $5(12.2)$ & $3(4.8)$ & $8(9.8)$ & $14(17.3)$ \\
\hline Era $6(15.7 \%, 2009-2014)$ & $1(6.2)$ & $7(12.5)$ & $5(10.6)$ & $14(26.4)$ \\
\hline Total $(25.9 \% ; 1984-2014)$ & $160(27.0)$ & $62(21.8)$ & $87(20.8)$ & $117(32.6)$ \\
\hline
\end{tabular}

For purposes of this analysis, risk factors were presence of anomalous pulmonary venous drainage, moderate to severe atrioventricular valve regurgitation, confirmed/suspected genetic anomaly, intact/highly restrictive atrial septum, preterm birth (dichotomized at 37 weeks), and weight at stage 1 reconstruction (dichotomized at $2.5 \mathrm{~kg}$ ). 
year experience of the Norwood procedure in New Zealand was reported by $\mathrm{Oh}$ and colleagues. ${ }^{12}$ For this 133 patient cohort, hospital mortality was $17 \%$ and ascending aortic size was identified as a risk factor for death.

\section{Study Limitations}

The primary limitations of this study are its retrospective nature and changing methods for risk factor ascertainment and documentation over time. Methodology for genetic testing and the use of genetic testing have changed markedly. Only paper charts are available for the earliest eras, and often the complete records could not be located. In addition, over the 30-year period, there have been many changes in prenatal care, perioperative care, surgical techniques, and bypass technology, which were not assessed during early eras but likely affected mortality. We did not examine the impact of bypass strategies or operative management factors.

\section{CONCLUSIONS}

Operative mortality for the Norwood procedure has been dramatically reduced since its introduction in 1983. However, early outcomes have plateaued over the last 10 to 15 years. Despite dramatic improvements in care, operative mortality for the Norwood procedure remains high compared with that for other forms of congenital heart disease. Our data suggest that much of the risk for early mortality is due to underlying nonmodifiable patient characteristics.

\section{Webcast}

You can watch a Webcast of this AATS meeting presentation by going to: https://aats.blob.core.windows.net/media/ 18Apr30/20ABC \% 201.Plenary \%20Sessions/S59\%20-\% 20Part\%202/S59_2.mp4.

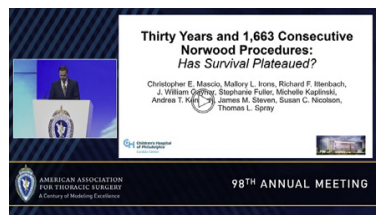

\section{Conflict of Interest Statement}

C.E.M. is a clinical consultant to HeartWare. All other authors have nothing to disclose with regard to commercial support.

\section{References}

1. Lev M. Pathologic anatomy and interrelationship of hypoplasia of the aortic tract complexes. Lab Invest. 1952;1:61-70.

2. Noonan JA, Nadas AS. The hypoplastic left heart syndrome; an analysis of 101 cases. Pediatr Clin North Am. 1958;5:1029-56.

3. Doty DB, Knott HW. Hypoplastic left heart syndrome. Experience with an operation to establish functionally normal circulation. J Thorac Cardiovasc Surg. 1977;74:624-30.
4. Norwood WI, Lang P, Hansen DD. Physiologic repair of aortic atresiahypoplastic left heart syndrome. N Engl J Med. 1983;308:23-6.

5. Tabbutt S, Ghanayem N, Ravishankar C, Sleeper LA, Cooper DS, Frank DU, et al. Risk factors for hospital morbidity and mortality after the Norwood procedure: a report from the Pediatric Heart Network Single Ventricle Reconstruction trial. J Thorac Cardiovasc Surg. 2012;144:882-95.

6. Wilder TJ, McCrindle BW, Hickey EJ, Ziemer G, Tchervenkov CI, Jacobs ML, et al. Is a hybrid strategy a lower-risk alternative to stage 1 Norwood operation? J Thorac Cardiovasc Surg. 2017;153:163-72.e6.

7. Baba K, Kotani Y, Chetan D, Chaturvedi RR, Lee KJ, Benson NL, et al. Hybrid versus Norwood strategies for single-ventricle palliation. Circulation. 2012; 126(11 Suppl 1):S123-31.

8. Mahle WT, Spray TL, Wernovsky G, Gaynor JW, Clark BJ III. Survival after reconstructive surgery for hypoplastic left heart syndrome: a 15-year experience from a single institution. Circulation. 2000;102(19 Suppl 3):III136-41.

9. Gaynor JW, Mahle WT, Cohen MI, Ittenbach RF, DeCampli WM, Steven JM, et al. Risk factors for mortality after the Norwood procedure. Eur J Cardiothorac Surg. 2002;22:82-9.

10. Alsoufi B, Mori M, Gillespie S, Schlosser B, Slesnick T, Kogon B, et al. Impact of patient characteristics and anatomy on results of Norwood operation for hypoplastic left heart syndrome. Ann Thorac Surg. 2015;100:591-8.

11. Kalfa D, Krishnamurthy G, Levasseur S, Najjar M, Chai P, Chen J, et al. Norwood stage I palliation in patients less than or equal to $2.5 \mathrm{~kg}$ : outcomes and risk analysis. Ann Thorac Surg. 2015;100:167-73.

12. Oh TH, Artrip JH, Graddon C, Minogue C, Marcondes L, Finucane K, et al. The New Zealand Norwood procedure experience: 22-year cumulative review. Heart Lung Circ. 2017;26:730-5.

Key Words: hypoplastic left heart syndrome, Norwood procedure, outcomes

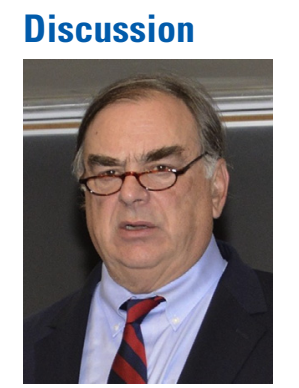

Dr M. Jacobs (Baltimore, Md). The Norwood procedure, the most commonly performed open operation in the neonatal age group, was developed approximately 40 years ago by Dr William Norwood. This operation has probably been the subject of as many or more investigations or reports than any other operation for congenital heart disease, yet Dr Mascio and colleagues stated accurately in their article that the principles of the Norwood operation remain essentially the same today as when Norwood first conceived it. This, of course, attests to the soundness of Norwood's successful concept for initial palliation for hearts with functionally univentricular anatomy and ductal dependence of the systemic circulation.

Dr Mascio and associates reported on a 30-year experience with the Norwood operation in Philadelphia and share with us this important report. Based on their analysis of this rich, unique dataset, they reached 2 fundamental conclusions. First, although hospital survival has improved dramatically since the introduction of the Norwood operation more than 35 years ago, it appears to have plateaued in the last 10 to 15 years. Second, much of the risk for early 
mortality is related to underlying patient characteristics, and many of these may not be modifiable.

Dr Mascio, you chose a novel and detailed 3-tiered approach to statistical analysis with adjustment for era at each stage. Apart from the striking and important, but not surprising effect of era of surgery, the final multiple covariate model identified only anomalous pulmonary venous connection, lower gestational age, and nonwhite, non-African-American race as being independently associated with increased mortality. Previously identified risk factors, such as intact or highly restrictive atrial septum, were not significant on multivariable analysis. Some factors appreciated in other studies, such as the presence of noncardiac anatomic abnormalities, were not included in your analysis.

Apart from the decision to not consider certain patient characteristics, such as the presence of noncardiac anatomic abnormalities, if one were to shed light on some of the intrinsic patient characteristics that are present at the time of birth that could have an impact on operative mortality, he would design the study pretty much as you did. If, on the other hand, the goal was to assess the importance of a broad spectrum of patient factors present before surgery, not necessarily at the time of birth, one would include factors such as circulatory collapse and organ system dysfunction secondary to ductal constriction, preoperative ventilatory support or pharmacologic support, and even the preoperative function of the systemic single ventricle.

I am grateful to you for explaining to me that you chose to intentionally exclude from analysis patient factors that could, to some extent, be influenced by processes of care. These then are relegated to the status of potential confounders that are not accounted for. This in turn leads to your almost inescapable conclusion that patient characteristics, some of which may not be modifiable, are principal determinants of mortality risk. It seems that you are inferring that your observed hospital mortality of $14 \%$ over the latter 15 -year period represents a plateau imposed by patient characteristics that are largely unmodifiable.

The discussion of many of the surgical articles this morning focused on technical aspects of surgery. I am going to ask you a question and hope that you will give an answer that is a bit more philosophical in nature. The aggregate unadjusted Norwood mortality rate across a recent 4-year period and encompassing approximately 100 centers in the Society of Thoracic Surgeons Congenital Heart Surgery Database was $14.7 \%$, not significantly different from the $14 \%$ average mortality over 15 years in your report. Does this then suggest that we have all hit the wall and that we have all hit the wall at about the same time? Is this as good as it gets? Or, alternatively, if one chooses to be more optimistic, then where should our energies and attention be focused to depart from this plateau and bring about further improvements in operative mortality for these patients?

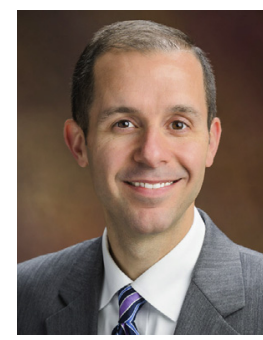

Dr Christopher F. Mascio (Philadelphia, $P a$ ). I think we have hit the wall somewhat, but I would like to think that it is not permanent. We have certainly gotten better over this 30-year period; milrinone and better intensive care unit care have definitely brought this rate down. One point we did want to make is that we essentially perform the same operation that was done back in 1984. There have been a few modifications.

Where should we focus our efforts? We need to look and see if we can change some of these factors that we can modify: the artificial womb that is being developed at Children's Hospital of Philadelphia, for example, and gene therapies. I am speaking in general terms, but these are the areas that we need to examine if we want to make this even better.

Dr Jacobs. That brings me to my second question. I tend to believe that some of these seemingly unmodifiable risk factors may be modifiable in some ways. For example, I want to ask you about prenatal diagnosis. Prenatal diagnosis, of course, offers the possibility that gestational age at birth may be a modifiable factor. With the recent understanding that a difference of 1 week in gestational age at birth, for example, between 37 and 38 weeks or between 35 and 36 weeks, with sophisticated prenatal, fetal, maternal, and cardiac coordination of care may affect outcomes, isn't it possible that we can and perhaps should now focus our efforts on "modifying" gestational age at birth, which, in your analysis, was treated as an unmodifiable factor? Your colleague at Children's Hospital of Philadelphia, Dr Michael Quartermain, recently completed an analysis of the Society of Thoracic Surgeons Database data that showed that in a contemporary multicenter cohort approximately $75 \%$ of patients with HLHS have a prenatal diagnosis. Furthermore, the investigation confirmed that neonates with HLHS who had a prenatal diagnosis had a lower prevalence of major preoperative comorbidities and risk factors compared with neonates without a prenatal diagnosis.

What was the frequency of prenatal diagnosis in the earlier era and the latter era? Perhaps more important, over this 15 -year period of "plateau" in outcomes, has there been a change in the prevalence and the practice of prenatal diagnosis in your center?

Dr Mascio. I think that number has gone up. I don't have the exact number, but I think that in the article you reference by Mike, it's in the $65 \%$ to $80 \%$ range. It's certainly more than it was back in the first 15 years of the study. That could be something that we can intervene on, as you say. There aren't many articles suggesting that prenatal diagnosis changes outcomes. I think things like prostaglandins are present at most hospitals and for children who are born at outside hospitals; generally, these things can get picked up and taken care of quite well.

Dr Jacobs. My final question goes to the ingenious and informative way that you looked at the cumulative burden 
of risk factors and their impact on mortality over time. But it harkens back to one of the points of Professor Martin Elliott's elegant presentation during this meeting. The challenge of going back 30 years and collecting the data is immense, and as you pointed out candidly in your slides and in the article, there were early eras when data completeness was $77 \%$, whereas for the most recent eras it was $100 \%$. As a result, ascertainment of many relevant data elements may be better, that is, more complete in the recent era than for earlier eras. Because this is likely true with respect to genetic anomalies, gestational age, and things like that, do you have any suspicion that maybe being identified as having 3 "risk factors" in the more recent era is kind of similar to being identified as having 2 factors in the earlier era because ascertainment wasn't nearly as good or as precise?

Dr Mascio. I think so. There are a couple of other things that could speak to that. The way the slides were set up made it look like the earlier eras had fewer risk factors, but I would guess that in the earlier eras the high-risk kids probably didn't make it. So, a larger proportion of those patients in the earlier eras had zero or 1 or 2 risk factors because the kids they had were sicker and probably didn't survive. Back at that time, the Children's Hospital of Philadelphia was pretty much the only place where this operation was performed, so they got everybody, whereas today most institutions would certainly hold on to their own patients with HLHS who had zero or 1 or 2 risk factors.

Dr Jacobs. Chris, I congratulate you on this enormous and successful undertaking, and I commend you and your colleagues on their huge efforts and enormous achievements on behalf of all these children and their families. It is really monumental.

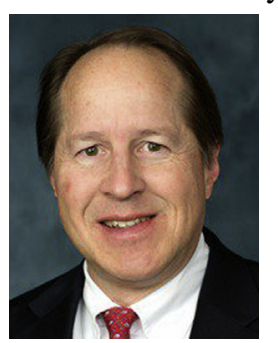

Dr Carl L. Backer (Chicago, Ill). Chris, it is fantastic to hear this follow-up. I remember coming to this meeting when people were presenting 10 or 15 Norwood operations, and we thought those were large series!

I have 2 questions for you. First, when the Australians looked at their results with tetralogy of Fallot, they looked at the outcomes of individual surgeons. They did not identify the surgeons, but they had charts that showed the freedom from reoperation for each surgeon's cohort of tetralogy of Fallot cases. Did you by any chance look at the outcomes of individual surgeons? I saw there were about 8 surgeons who did the Norwood operations. Was there any difference among the surgeons? You may or may not have those data.

Dr Mascio. We don't have those data.

Dr Backer. I think that would be an interesting thing to look at. The second question relates to the debate between use of the modified Blalock-Taussig shunt versus the right ventricle-pulmonary artery (RV-PA) conduit, or Sano shunt; Dr Sano is right behind me. Did you have an analysis of those data, and was there any difference between those 2 groups?

Dr Mascio. We do have those data. This is just scratching the surface of this project, which we hope will provide many more presentations. With this presentation, we just focused on patient risk factors.

My comment about the RV-PA shunt was based on Sarah Tabbutt's article with the Single Ventricle Randomization trial that showed in-hospital mortality was the same for the BT shunt versus the RV-PA shunt. Because we were only looking at those who survived the hospital, we thought that that was good to include. But we hope that further talks and article will shed light on operative factors, how many kids survived to the Glenn, how many kids survived the Fontan, and so forth.

Dr Shunji Sano (Okayama, Japan). My experience is very small, we are doing 5 to 10 cases a year, but we analyzed our data 15 years after I started the RV-PA shunt with 100 cases. The mortality is high, the same as yours, compared with $5 \%$ in normal-risk patients. We thought that some of the risk factors could be excluded, such as low birth weight, prematurity, and restrictive atrial septal defect if we enlarge the atrial septal defect before actual stage I palliation. Therefore, we perform a rapid 2-stage followed by bilateral PA banding until the patient's body weight increases more than 2.5 or gestational age to reach normal. Then our results have improved. You analyzed a huge study and know the high risk. Is there any new strategy to improve the result in the future from your analysis?

Dr Mascio. From this analysis, the comment I made to Dr Jacobs about artificial wombs, so that kids aren't born early, gene therapy, and so forth, perhaps will provide a platform. I think you are right. In the last 10 years at Children's Hospital of Philadelphia, if you have zero to 2 risk factors, you have a $0 \%$ to $5 \%$ mortality. I think all of us have gotten quite good when there are minimal risk factors. But it's really hard to change the size of a child's ascending aorta, sometimes kids have to be born early. It is certainly a challenge.

Dr Sano. In relation to our rapid 2-stage strategy, I started stem cell therapy to the hypoplast and the single ventricle and to the patient with poor ventricular function. I must say, the median ejection fraction improvement in 5 years is $9 \%$.

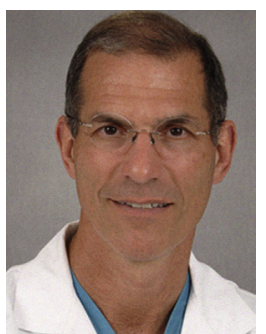

Dr Glenn Whitman (Baltimore, $M d$ ). As everybody knows, I know nothing about pediatric heart surgery, but I was intrigued by Marshall's analysis of what's going on here and the plateauing mortality, and that maybe we have hit the wall. I wondered whether there was a chance here to implement Frank Shannon's phase of care analysis for mortality that is going on in Michigan where they look at every death in the state and try to pick where 
in the patient's care the mistake was made that led to a mortality and whether that mistake was preventable. And if you applied that analysis to these patients who die, do you think that one could learn something and perhaps inch down on the mortality?

Dr Mascio. I am not so sure, to be honest. I think most of us have learned how to do this operation, and even if there is PA stenosis or shunt stenosis, that is nothing that a stent can't help. Often, we have a lot of kids who don't survive, and their last echo showed that the atrial septum is wide open, the function was adequate, the arch was unobstructed, and they had adequate saturations. I'm not saying that you wouldn't pick up 1 or 2 patients, but I don't know that that would make a huge impact on this lesion today.

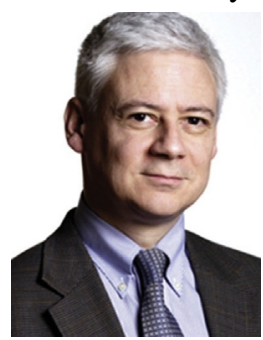

Dr Christian Pizarro (Wilmington, Del). Chris, a beautiful presentation and robust data set. It would be helpful for all of us to know some of the trajectory of your practice at your institution: How did hybrid procedures inform the choice of intervention in these patients, how did the Single
Ventricle Randomization trial influence, if at all, your practice, and how do you select what type of shunt for any particular patient?

Dr Mascio. We rarely use the hybrid procedure, only for very sick kids, really bad function, severe aortic valve regurgitation, and end-organ dysfunction. As far as what type of shunt we use, we have pretty much gone to the RV-PA shunt, even in patients with aortic stenosis, to be honest, unless it's a variant with anterior-posterior great vessels, and we are forced to do a Blalock-Taussig shunt. But we have pretty much gone to the RV-PA shunt.

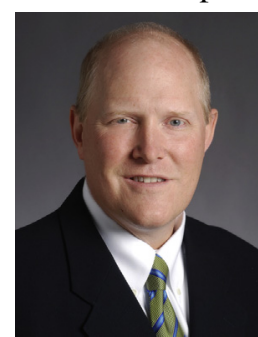

Dr James S. Tweddell (Cincinnati, Ohio). A great study. Sort of to Marshall's point about perhaps early in the experience not identifying all the risk factors, I was struck that female sex was a borderline risk factor. Do you think that might be accounted for by undiagnosed Turner syndrome among that population?

Dr Mascio. I guess it's possible. I don't know what that percentage is in those who have HLHS, but I suppose it's possible. 